\title{
A data-driven approach to stochastic constrained control of piecewise affine systems
}

\author{
Riccardo Maria Vignali ${ }^{1}$, Daniele Ioli $^{2}$, Maria Prandini ${ }^{2}$
}

\begin{abstract}
This paper addresses the design of the control input to a discrete time piecewise affine system so as to optimize its performance along a finite time horizon. The system is affected by some additive stochastic disturbance and is subject to constraints that need to be appropriately accounted for in the design. By enforcing constraints to be satisfied for almost all disturbance realizations except for a set of probability $\epsilon$, we can decide to what extent trading robustness for improving performance and also cope with the case of disturbance with unbounded support. Inspired by the existing literature, we propose a method to realize this trade-off, which combines the scenario approach to chance-constrained optimization with robust optimal control of piecewise affine systems. Interestingly, no explicit knowledge of the disturbance distribution is needed but only some disturbance realizations, thus resulting in a datadriven design method.
\end{abstract}

\section{INTRODUCTION}

In this paper, we address control input design for a discrete time PieceWise Affine (PWA) system affected by a stochastic disturbance, possibly with unbounded support. The probabilistic characteristics of the disturbance are known indirectly, thought historical data, and the goal is to optimize the controlled system performance over a finite time horizon, while satisfying state and actuation constraints.

PWA systems are characterized by a polyhedral partition of the state cross (control and disturbance) input space. Each element in the partition is a mode and has associated a certain affine dynamics. The mode - and, hence, the corresponding affine dynamics - is active when the state and input belong to that element of the partition.

The class of PWA systems have been extensively studied in the literature, with reference to the analysis of properties like controllability and observability, and problems like model reduction and minimal realization, identification, fault detection and estimation, stabilization, and control, see, e.g., [1, Chapters 4 and 5]. This is partly motivated by the fact that methods from linear systems theory can be adapted to a PWA modeling framework. Moreover, various systems are naturally described as PWA systems and nonlinear systems can be approximated up to a desired accuracy level with PWA models by means of suitable hybridization procedures (see, e.g., [2]-[5]). In robust control and verification, the

This work is partially supported by the European Commission under the project UnCoVerCPS with grant number 643921.

${ }^{1}$ R.M. Vignali is with RSE Spa, Ricerca sul Sistema Energetico, Via Rubattino 54, 20124 Milano, Italy. riccardo.vignaliarse-web.it

${ }^{2}$ D. Ioli and M. Prandini are with Dipartimento di Elettronica, Informazione e Bioingegneria, Politecnico di Milano, via Ponzio 34/5, 20133 Milano, Italy. \{daniele.ioli, maria.prandini\} apolimi.it introduced approximation error can be accounted for as a fictitious additive disturbance so that the obtained controller performance or the outcome of the verification are guaranteed, although conservative. From a computational viewpoint, verification and control design problems for PWA systems can be formulated as Mixed Integer Linear or Quadratic Programming (MILP/ MIQP) problems, since PWA systems are described by affine equations and inequalities, and mode switching can be described by introducing integer variables. The resulting optimization problems are NP hard to solve, but many algorithms and software tools exist to tackle them. This is in essence what is done when PWA systems are converted into Mixed Logical and Dynamical (MLD) systems, [6]-[8], which are equivalent to PWA systems under suitable wellposedness conditions, [9].

Here, we are concerned with a PWA system that is subject to a possibly unbounded disturbance, and address the design of the control input that minimizes a given cost function while satisfying actuation and state constraints. The disturbance is stochastic and its probability distribution is not known explicitly but only indirectly via some independent realizations extracted at random from such a distribution, which calls for a data-driven approach. Due to the state constraints and the fact that the disturbance is possibly unbounded, we cannot address the problem according to a worst case, $100 \%$ robust, perspective where constraints must hold for every and each disturbance realization. We instead formulate the problem according to a probabilistic perspective, where state constraints are enforced and performance is optimized over a set of disturbance realizations of probability $1-\epsilon$, with the parameter $\epsilon \in(0,1)$ setting the appropriate compromise between performance and robustness. The resulting chance-constrained optimization program is hard to be solved, also because of the PWA dynamics. We then tackle it via the two step procedure suggested in [10], where the disturbance is first confined to a compact set of probability $1-\epsilon$, and then a robust optimization problem is formulated over the obtained compact set. As for the first step, we resort to the so-called scenario approach, [11]-[13], to define a box of probability $1-\epsilon$ for the disturbance. Remarkably, this approach requires only to know disturbance realizations (scenarios) in order to compute the box, thus allowing for a data-driven methodology. As for the second step, we show that, if the cost function is linear in the state and control input, and the state constraints are polytopic, then, the resulting robust optimization problem for the PWA system subject to the box disturbance can be reduced to a MILP problem. This is achieved via a geometric interpretation 
of the robust constraints as a set-containment condition as suggested in [14] in the context of PWA systems testing and verification. In [15], a similar robust optimization problem is addressed but for linear systems and an LMI (Linear Matrix Inequalities) reformulation is adopted. In [16], robust control for PWA systems is performed but based on some robust mode control restriction, [17], that makes the mode sequence of the controlled system independent of the disturbance realization.

The rest of the paper is organized as follows. We describe the problem addressed and the resulting chance-constrained optimization program formulation in Section II. In Section III a data-driven MILP solution to the chance-constrained optimization program is worked out by integrating scenario and robust optimization. Section IV draws some concluding remarks and outline further research directions.

\section{PROBlEM FORMULATION}

We consider a discrete time system with state $x \in \mathbb{R}^{n}$ and control input $v \in \mathbb{R}^{m_{v}}$, which is affected by some stochastic disturbance $w \in \mathbb{R}^{m_{w}}$ with a possibly unbounded support. The state of the system evolves according to a PieceWise Affine (PWA) dynamics, that is its dynamics is affine and given by

$$
x(k+1)=A_{i} x(k)+B_{i}^{v} v(k)+B_{i}^{w} w(k)+f_{i},
$$

when $\left[x^{\prime}(k) v^{\prime}(k) w^{\prime}(k)\right]^{\prime} \in \mathcal{A}_{i}$, where $A_{i}, B_{i}^{v}$, and $B_{i}^{w}$ are matrices of appropriate dimension, $f_{i}$ is a constant vector, and $\mathcal{A}_{i}$ is a polyhedron of dimension $n+m_{v}+m_{w}$ described by

$$
\mathcal{A}_{i}=\left\{(x, v, w):\left[\begin{array}{lll}
L_{a x}^{i} & L_{a v}^{i} & L_{a w}^{i}
\end{array}\right]\left[\begin{array}{lll}
x^{\prime} & v^{\prime} & w^{\prime}
\end{array}\right]^{\prime} \leq L_{b}^{i}\right\},
$$

where $L_{a x}^{i}, L_{a v}^{i}, L_{a w}^{i}$, and $L_{b}^{i}$ define the H-representation of $\mathcal{A}_{i}$ as an intersection of half-spaces, [18], $i=1,2, \ldots, s$. The collection $\{\mathcal{A}\}_{i=1}^{s}$ constitutes a polyhedral subdivision of the space $\mathbb{R}^{n} \times \mathbb{R}^{m_{v}} \times \mathbb{R}^{m_{w}}$, i.e., $\cup_{i=1}^{s} \mathcal{A}_{i}=\mathbb{R}^{n} \times \mathbb{R}^{m_{v}} \times \mathbb{R}^{m_{w}}$ and the intersection $\mathcal{A}_{i} \cap \mathcal{A}_{j}, i \neq j$, is either empty or a common proper face of both polyhedra. Polyhedron $\mathcal{A}_{i}$ is called mode $i$, and mode $i$ is active at time $k$ if $x(k) \in \mathcal{A}_{i}$. A numerical test for checking if the resulting PWA system is well posed (its evolution is always well defined) can be found in [8]. Since the modes define a partition of the stateinput-disturbance space, there is only one mode active at a time.

Our goal is designing the control input vector over a time horizon of finite length $T: \boldsymbol{v}=\left[v(0)^{\prime} v(1)^{\prime} \ldots v(T-1)^{\prime}\right]^{\prime} \in$ $\mathbb{R}^{m_{v} T}$ so as to minimize the linear cost function

$$
J(\boldsymbol{v})=\sum_{k=0}^{T-1}\left(c_{x}^{\prime}(k+1) x(k+1)+c_{v}^{\prime}(k) v(k)\right),
$$

when the system is initialized at $x(0)=x_{0}$, subject to the actuation constraints on the input $v(k) \in\left[\underline{v}_{k}, \bar{v}_{k}\right] \subset \mathbb{R}^{m_{v}}$, and (affine) state constraints of the form $x(k) \in \mathcal{X}_{k}, k=$ $1,2, \ldots, T$, where $\mathcal{X}_{k}$ is a polytopic set described by

$$
\mathcal{X}_{k}=\left\{x \in \mathbb{R}^{n}: H_{a}^{k} x \leq H_{b}^{k}\right\}
$$

with $H_{a}^{k}$ and $H_{b}^{k}$ denoting the matrices of the $H$ representation of $\mathcal{X}_{k}, k=1,2, \ldots, T$.

Note that the cost $J$ in (3) is actually uncertain since it depends on the disturbance vector

$$
\boldsymbol{w}=\left[w(0)^{\prime} w(1)^{\prime} \ldots w(T-1)^{\prime}\right]^{\prime} \in \mathbb{R}^{m_{w} T}
$$

through the state evolution $x(k+1), k=0, \ldots, T-1$, which is obtained via the PWA dynamics (1) initialized with $x(0)=x_{0}$. To recall this dependence on $\boldsymbol{w}$, we shall use the notation $J_{\boldsymbol{w}}$ and $x_{\boldsymbol{w}}$ when needed.

In order to take into account the uncertainty $\boldsymbol{w}$, we adopt a probabilistic perspective and impose that an upper bound $\ell$ on the cost (3) is minimized and constraints are satisfied for all disturbance realizations except for a set of a priori defined probability $\epsilon \in(0,1)$. More precisely, we address the following problem

$$
\min _{\boldsymbol{v} \in \mathcal{V}, \ell} \ell
$$

subject to:

$$
\mathbb{P}_{\boldsymbol{w}}\left\{J_{\boldsymbol{w}}(\boldsymbol{v}) \leq \ell \wedge x_{\boldsymbol{w}}(k) \in \mathcal{X}_{k}, k=1,2 \ldots T\right\} \geq 1-\epsilon,
$$

where $\mathbb{P}_{\boldsymbol{w}}$ is the probability induced on the system trajectories by the uncertainty $\boldsymbol{w}$, and $\mathcal{V}$ is the box with lower and upper bounds $\underline{\boldsymbol{v}}=\left[\begin{array}{llll}\underline{v}_{0}^{\prime} & \underline{v}_{1}^{\prime} & \ldots & \underline{v}_{T-1}^{\prime}\end{array}\right]^{\prime}$ and $\overline{\boldsymbol{v}}=\left[\begin{array}{llll}\bar{v}_{0}^{\prime} & \bar{v}_{1}^{\prime} & \ldots & \bar{v}_{T-1}^{\prime}\end{array}\right]^{\prime}$, respectively, that is, $\mathcal{V}=[\underline{\boldsymbol{v}}, \overline{\boldsymbol{v}}]=$ $\left[\underline{v}_{0}, \bar{v}_{0}\right] \times\left[\underline{v}_{1}, \bar{v}_{1}\right] \times \cdots \times\left[\underline{v}_{T-1}, \bar{v}_{T-1}\right]$. The minimizer $\left(\boldsymbol{v}^{\star}, \ell^{\star}\right)$ will provide the control input $\boldsymbol{v}^{\star} \in \mathcal{V}$ that guarantees the best (lowest) value $\ell^{\star}$ for $J_{\boldsymbol{w}}(\boldsymbol{v})$ over all disturbance realizations except for a set of probability at most $\epsilon$ while satisfying the state constraints.

It is worth noticing that the violation probability $\epsilon$ plays the role of a tuning knob trading robustness for performance in that if we increase $\epsilon$ we improve performance, but decrease the guarantees that such a performance and also the state constraints are satisfied. Depending on the application at hand, $\epsilon$ can be appropriately set so as to get the desired compromise and set the risk at some acceptable value.

Note that not all values for $\epsilon$ may be admissible for the chance-constrained problem (3) to be feasible. This is for instance the case when $w$ has an unbounded support, and the state is confined to some compact set $\mathcal{X}_{k}$ that is not compatible with the available actuation effort and the experienced extent of the disturbance $w$. In the following we shall neglect this issue and assume feasibility. The interested reader can refer to [19] for an effective solution to the state feasibility issue, which could be adopted and captured in our framework, but that we do not take into account here to simplify the presentation of our approach.

\section{PROBLEM SOLUtion}

The chance-constrained optimization program (5) is generally hard to solve except for a few cases like when probability $\mathbb{P}_{\boldsymbol{w}}$ is Gaussian, [20], [21]. It is indeed even to solve in a data-driven framework, where $\mathbb{P}_{\boldsymbol{w}}$ is not known explicitly but only indirectly through some independent extractions $\boldsymbol{w}^{(1)}, \boldsymbol{w}^{(2)}, \ldots, \boldsymbol{w}^{(N)}$ of $\boldsymbol{w}$ (scenarios). 
Following [10], we then propose a two step data-based solution, where we first determine a compact set $\mathcal{W}$ for vector $\boldsymbol{w}$ such that $\mathbb{P}_{\boldsymbol{w}}\{\boldsymbol{w} \in \mathcal{W}\} \geq 1-\epsilon$, and we then solve the following robust optimization problem

$$
\min _{\boldsymbol{v} \in \mathcal{V}, \ell \in \mathcal{L}} \ell
$$

subject to:

$$
J_{\boldsymbol{w}}(\boldsymbol{v}) \leq \ell \wedge x_{\boldsymbol{w}}(k) \in \mathcal{X}_{k}, k=1,2 \ldots T, \boldsymbol{w} \in \mathcal{W},
$$

$\mathcal{L}$ being the compact interval where $\ell$ can be confined given that the system (1) is initialized at $x_{0}$ and subject to compact inputs $\boldsymbol{w} \in \mathcal{W}$ and $\boldsymbol{v} \in \mathcal{V}$.

Given that probability $\mathbb{P}_{\boldsymbol{w}}$ is known only indirectly, through the collected scenarios, the first step is addressed via randomization and an appropriate convex problem formulation so as to guarantee that $\mathbb{P}_{\boldsymbol{w}}\{\boldsymbol{w} \in \mathcal{W}\} \geq 1-\epsilon$ with a certain (high) confidence $1-\beta, \beta \in(0,1)$. The solution to the robust program (6), then, will inherit the probabilistic guarantees of $\mathcal{W}$, i.e., it will be feasible for the original chance-constrained program (5) with confidence $1-\beta$.

Note that we adopt a two step approach since the scenario guarantees do not hold for the randomized version of the original chance-constrained problem (5), because constraints inside $\mathbb{P}_{\boldsymbol{w}}$ are not convex in the optimization variable $\boldsymbol{v}$ due to the PWA dynamics of the system.

We next explain in detail how the two steps are performed.

\section{Step 1: bounding uncertainty via scenario optimization}

Set $\mathcal{W}$ is chosen to be a box

$$
\mathcal{W}=[\underline{\boldsymbol{w}}, \overline{\boldsymbol{w}}],
$$

with $\underline{\boldsymbol{w}}=\left[\begin{array}{llll}\underline{w}_{0} & \underline{w}_{1} & \ldots & \underline{w}_{T-1}\end{array}\right]^{\prime}$ and $\overline{\boldsymbol{w}}=\left[\begin{array}{llll}\bar{w}_{0} & \bar{w}_{1} & \ldots & \bar{w}_{T-1}\end{array}\right]^{\prime}$.

The problem of determining $[\underline{\boldsymbol{w}}, \overline{\boldsymbol{w}}]$ based on the available scenarios $\left\{\boldsymbol{w}^{(i)}\right\}_{i=1}^{N}$ is addressed by solving the following scenario optimization program

$$
\begin{aligned}
& \min _{\overline{\boldsymbol{w}}, \underline{\boldsymbol{w}}} \sum_{h=0}^{T-1}\left\|\bar{w}_{h}-\underline{w}_{h}\right\| \\
& \text { subject to: } \\
& \boldsymbol{w}^{(i)} \in[\underline{\boldsymbol{w}}, \overline{\boldsymbol{w}}], \quad i=1,2, \ldots, N .
\end{aligned}
$$

The following proposition then can be directly derived from [10, Section III].

Proposition 1: Let $\left(\overline{\boldsymbol{w}}^{\star}, \underline{\boldsymbol{w}}^{\star}\right)$ be the solution to (7). If $N$ satisfies $(1+(N-1) \bar{\epsilon})(1-\bar{\epsilon})^{N-1} \leq \bar{\beta}$, where $\bar{\epsilon}=\frac{\epsilon}{T}$ and $\bar{\beta}=\frac{\beta}{T}$, then, it holds that $\mathbb{P}_{\boldsymbol{w}}\left\{\boldsymbol{w} \in\left[\underline{\boldsymbol{w}}^{\star}, \overline{\boldsymbol{w}}^{\star}\right]\right\} \geq 1-\epsilon$, with confidence larger than or equal to $1-\beta$.

\section{Step 2: control input design via robust optimization}

We start by better formulating problem (6), making the constraints that need to be robustly satisfied explicit as a function of state and input variables:

$$
\min _{v \in \mathcal{V}, \ell \in \mathcal{L}} \ell
$$

subject to:

$$
\begin{aligned}
& \sum_{k=0}^{T-1}\left(c_{x}^{\prime}(k+1) x(k+1)+c_{v}^{\prime}(k) v(k)\right) \leq \ell \\
& H_{a}^{k} x(k) \leq H_{b}^{k}, \quad k=1,2, \ldots T, \\
& x(k+1)=A_{i} x(k)+B_{i}^{v} v(k)+B_{i}^{w} w(k)+f_{i}, \\
& \text { for }\left[x^{\prime}(k) v^{\prime}(k) w^{\prime}(k)\right]^{\prime} \in \mathcal{A}_{i}, k=0, \ldots, T-1, \\
& \boldsymbol{w} \in \mathcal{W} .
\end{aligned}
$$

According to (1) initialized with $x(0)=x_{0}$, the state $x(k)$ can be expressed as follows:

$$
\begin{aligned}
x(k)= & \prod_{j=0}^{k-1} A_{i(j)} x_{0}+\sum_{j=0}^{k-1}\left(\left(\prod_{h=j+1}^{k-1} A_{i(h)}\right) B_{i(j)}^{v} v(j)\right. \\
& \left.+\left(\left(\prod_{h=j+1}^{k-1} A_{i(h)}\right) B_{i(j)}^{w} w(j)\right)+f_{i(j)}\right),
\end{aligned}
$$

where $i(j) \in\{1, \ldots, s\}$ is the index of the mode active at time $j$. The ordered collection of modes $I=$ $\{i(0), i(1), \ldots, i(k-1)\} \in\{1, \ldots, s\}^{k}$ that are active during the system evolution up to time $k$ is called switching sequence of the PWA system in the horizon $[0, k]$.

A solution to (8) exists if we can find $(\tilde{\boldsymbol{v}}, \tilde{\ell}) \in \mathcal{V} \times \mathcal{L}$ such that the set of states that the system can reach within the interval $[1, T]$ when its control input $\boldsymbol{v}$ is set equal to $\tilde{\boldsymbol{v}}$ is contained within the specified sets $\mathcal{X}_{k}, k=1,2 \ldots, T$ and $J(\tilde{\boldsymbol{v}}) \leq \tilde{\ell}$, when the disturbance $\boldsymbol{w}$ ranges in $\mathcal{W}$. Once we have characterized such feasible values $(\tilde{\boldsymbol{v}}, \tilde{\ell})$, we can then pick up one with the minimum value of $\tilde{\ell}$, thus solving the robust optimization problem (8).

The question is then how we shall characterize the feasible values $(\tilde{\boldsymbol{v}}, \tilde{\ell})$. We next suggest a geometric perspective resting on a set inclusion condition. To this purpose we first introduce some compact notations.

Set $m=m_{w}+m_{v}$, and define the input vector $u \in \mathbb{R}^{m}$ :

$$
u=\left[w^{\prime}, v^{\prime}\right]^{\prime} .
$$

Fix a switching sequence $I_{l}=\left\{i_{0}, i_{1}, \ldots, i_{T-1}\right\}$. The evolution of the state of system (1) on the time interval $[0, T]$

$$
X=\left[\begin{array}{lllll}
x(0)^{\prime} & x(1)^{\prime} & x(2)^{\prime} & \ldots & x(T)^{\prime}
\end{array}\right]^{\prime},
$$

starting from the initial condition $x(0)=x_{0}$ and subject to the input sequence

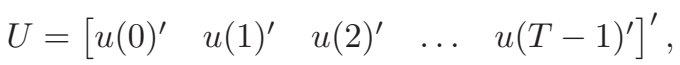

can be written as:

$$
X=\mathbf{B}^{\left(I_{l}\right)} U+\mathbf{G}^{\left(I_{l}\right)} F^{\left(I_{l}\right)}+\mathbf{A}^{\left(I_{l}\right)} x_{0},
$$

where we set

$$
\mathbf{B}^{\left(I_{l}\right)}=\left[\begin{array}{cccc}
0 & 0 & 0 & 0 \\
B_{i_{0}} & 0 & 0 & 0 \\
A_{i_{1}} B_{i_{0}} & B_{i_{1}} & 0 & 0 \\
\vdots & \vdots & \ddots & \vdots \\
\prod_{j=1}^{T-1} A_{i_{j}} B_{i_{0}} & \prod_{j=2}^{T-1} A_{i_{j}} B_{i_{1}} & \ldots & B_{i_{T-1}}
\end{array}\right],
$$


with $B_{i}=\left[\begin{array}{ll}B_{i}^{w} & B_{i}^{v}\end{array}\right]$,

$$
\begin{gathered}
\mathbf{G}^{\left(I_{l}\right)}=\left[\begin{array}{cccc}
0 & 0 & 0 & 0 \\
I & 0 & 0 & 0 \\
A_{i_{1}} & I & 0 & 0 \\
\vdots & \vdots & \vdots & \vdots \\
\prod_{j=1}^{T-1} A_{i_{j}} & \prod_{j=2}^{T-1} A_{i_{j}} & \ldots & I
\end{array}\right], \\
F^{\left(I_{l}\right)}=\left[\begin{array}{c}
f_{i_{0}} \\
f_{i_{1}} \\
f_{i_{2}} \\
\vdots \\
f_{i_{T-1}}
\end{array}\right],
\end{gathered}
$$

The constraints on the state-control input-disturbance pairs to the modes activation in the switching sequence can be written in the following compact form:

$$
\mathbf{L}_{a x}^{\left(I_{l}\right)} X+\mathbf{L}_{a u}^{\left(I_{l}\right)} U \leq \mathbf{L}_{b}^{\left(I_{l}\right)}
$$

where

$$
\mathbf{L}_{a x}^{\left(I_{l}\right)}=\left[\begin{array}{cccc}
L_{a x}^{i_{0}} & & & 0 \\
& \ddots & & \vdots \\
& & L_{a x}^{i_{T}-1} & 0
\end{array}\right] \mathbf{L}_{a u}^{\left(I_{l}\right)}=\left[\begin{array}{ccc}
L_{a u}^{i_{0}} & & \\
& \ddots & \\
& & L_{a u}^{i_{T-1}}
\end{array}\right]
$$$$
\mathbf{L}_{b}^{\left(I_{l}\right)}=\left[L_{b}^{i_{0}{ }^{\prime}}, L_{b}^{i^{i^{\prime}}}, \ldots L_{b}^{i_{T-1}{ }^{\prime}}\right]^{\prime},
$$

with $L_{a u}^{i}=\left[\begin{array}{ll}L_{a v}^{i} & L_{a w}^{i}\end{array}\right]$. By plugging (13) in (14) we get:

$$
\mathbf{L}_{a x}^{\left(I_{l}\right)}\left(\mathbf{A}^{\left(I_{l}\right)} x_{0}+\mathbf{B}^{\left(I_{l}\right)} U+\mathbf{G}^{\left(I_{l}\right)} F^{\left(I_{l}\right)}\right)+\mathbf{L}_{a u}^{\left(I_{l}\right)} U \leq \mathbf{L}_{b}^{\left(I_{l}\right)},
$$

which becomes:

$$
\mathbf{M}_{a}^{\left(I_{l}\right)} U \leq \mathbf{M}_{b}^{\left(I_{l}\right)},
$$

where $\mathbf{M}_{a}^{\left(I_{l}\right)}=\mathbf{L}_{a x}^{\left(I_{l}\right)} \mathbf{B}^{\left(I_{l}\right)}+\mathbf{L}_{a u}^{\left(I_{l}\right)}$ and $\mathbf{M}_{b}^{\left(I_{l}\right)}=\mathbf{L}_{b}^{\left(I_{l}\right)}$ $\mathbf{L}_{a x}^{\left(I_{l}\right)} \mathbf{A}^{\left(I_{l}\right)} x_{0}-\mathbf{L}_{a x}^{\left(I_{l}\right)} \mathbf{G}^{\left(I_{l}\right)} F^{\left(I_{l}\right)}$.

From now on, we will say that system (1) evolves in the switching sequence $I_{l}$ if equation (15) is satisfied. We also define the reach-set associated with $I_{l}$ in the enlarged variables space $\mathcal{U} \times \mathcal{L}$ as follows

$$
\mathcal{A}_{l}=\left\{(U, \ell) \in \mathcal{U} \times \mathcal{L}: \mathbf{M}_{a}^{\left(I_{l}\right)} U \leq \mathbf{M}_{b}^{\left(I_{l}\right)}\right\},
$$

where $\mathcal{U}=[\underline{\boldsymbol{u}}, \overline{\boldsymbol{u}}]$ with $\underline{\boldsymbol{u}}=\left[\begin{array}{lllll}\underline{w}_{0}^{\prime} & \underline{v}_{0}^{\prime} & \ldots & \underline{w}_{T-1}^{\prime} & \underline{v}_{T-1}^{\prime}\end{array}\right]^{\prime}$ and

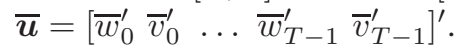

Let SS denote the set of all possible switching sequences. Then, we have that

$$
\bigcup_{I_{l} \in \mathbf{S S}} \mathcal{A}_{l}=\mathcal{U} \times \mathcal{L}
$$

since any value for $u(k)$ is admissible at each time step $k$, given that there always exists an active mode corresponding to that input value as dictated by the polyhedral subdivision defining the PWA dynamics.
The linear constraints in (9) and (10) can be rewritten as:

$$
\left\{\begin{array}{l}
C_{x}\left(\mathbf{A}^{\left(I_{l}\right)} x_{0}+\mathbf{B}^{\left(I_{l}\right)} U+\mathbf{G}^{\left(I_{l}\right)} F^{\left(I_{l}\right)}\right)+C_{u} U \leq \ell \\
H_{a}\left(\mathbf{A}^{\left(I_{l}\right)} x_{0}+\mathbf{B}^{\left(I_{l}\right)} U+\mathbf{G}^{\left(I_{l}\right)} F^{\left(I_{l}\right)}\right) \leq H_{b}
\end{array}\right.
$$

where $C_{x}=\left[\begin{array}{lllll}0 & c_{x}^{\prime}(1) & c_{x}^{\prime}(2) & \ldots & c_{x}^{\prime}(T)\end{array}\right], \quad C_{u}=$ $\left[\begin{array}{lllllll}0 & c_{v}^{\prime}(0) & 0 & c_{v}^{\prime}(1) & \ldots & 0 & c_{v}^{\prime}(T-1)\end{array}\right]$,

$$
H_{a}=\left[\begin{array}{cccc}
0 & H_{a}^{1} & & \\
\vdots & & \ddots & \\
0 & & & H_{a}^{T}
\end{array}\right], \quad H_{b}=\left[H_{b}^{1^{\prime}}, \ldots H_{b}^{T^{\prime}}\right]^{\prime} .
$$

We can rewrite the inequalities (17) in the compact form:

$$
\mathbf{S}_{a}^{\left(I_{l}\right)}\left[U^{\prime} \ell\right]^{\prime} \leq \mathbf{S}_{b}^{\left(I_{l}\right)},
$$

where $\mathbf{S}_{a}^{\left(I_{l}\right)}$ and $\mathbf{S}_{b}^{\left(I_{l}\right)}$ are suitably defined matrices, and introduce the spec-set

$$
\mathcal{S} \boldsymbol{p}_{l}=\left\{(U, \ell) \in \mathcal{U} \times \mathcal{L}: \mathbf{S}_{a}^{\left(I_{l}\right)}\left[U^{\prime} \ell\right]^{\prime} \leq \mathbf{S}_{b}^{\left(I_{l}\right)}\right\} .
$$

If $\mathcal{A}_{l} \cap \mathcal{S} p_{l}$ is not empty, its elements are input sequences $\{u(0), u(1), \ldots u(T-1)\}$ and values for $\ell$ that make the state of the system satisfies the constraints (9) and (10) while keeping its evolution in the switching sequence $I_{l}$.

Let $\tilde{z}=(\tilde{v}, \tilde{\ell}) \in \mathcal{V} \times \mathcal{L}$, and denote with $\Pi_{\tilde{z}}\left(\mathcal{A}_{l}\right)$ and $\Pi_{\tilde{z}}\left(\mathcal{S} \boldsymbol{p}_{l}\right)$ the slices of the reach-set $\mathcal{A}_{l}$ and spec-set $\mathcal{S} \boldsymbol{p}_{l}$ obtained by setting $\ell=\tilde{\ell}$ and fixing in $U$ (see (11) and (12)) the sequence $\{v(k)\}_{k=0}^{T-1}$ to the value $\tilde{v} \in \mathcal{V}$. Problem (8) is then feasible if there exist some value $\tilde{v}=\{v(k)\}_{k=0}^{T-1}$ for the control input and some value $\tilde{\ell}$ for the upper bound $\ell$ on the cost, such that the following condition holds:

$$
\Pi_{\tilde{z}}\left(\mathcal{A}_{l}\right) \subseteq \Pi_{\tilde{z}}\left(\mathcal{S p}_{l}\right) \quad \forall I_{l} \in \mathbf{S S} .
$$

This can be formulated as bilinear feasibility test, [22], which is hard to be solved. Here we head for an approximate MILP solution by over-approximating $\mathcal{A}_{l}$ by an outer box $\mathcal{B}_{l}$ so as to provide a sufficient condition for (20) to hold, i.e.,

$$
\Pi_{\tilde{z}}\left(\boldsymbol{B}_{l}\right) \subseteq \Pi_{\tilde{z}}\left(\boldsymbol{S} \boldsymbol{p}_{l}\right) \Rightarrow \Pi_{\tilde{z}}\left(\mathcal{A}_{l}\right) \subseteq \Pi_{\tilde{z}}\left(\boldsymbol{S} \boldsymbol{p}_{l}\right) .
$$

$\mathcal{B}_{l}$ will be chosen as the minimum volume outer box approximation of $\mathcal{A}_{l}$. Note that the over-approximations $\mathcal{B}_{i}$ and $\mathcal{B}_{j}$ of $\mathcal{A}_{i}$ and $\mathcal{A}_{j}$ associated to the switching sequences $I_{i} \neq I_{j}$ may partly overlap. In this case, the approach still remains valid though it is clearly conservative. The upper and lower limits $\bar{b}^{\left(I_{l}\right)}$ and $\underline{b}^{\left(I_{l}\right)}$ of $\mathcal{B}_{l}$ can be computed via LP as explained in [23] and $\mathcal{B}_{l}$ can be expressed as

$\boldsymbol{B}_{l}=\left[\underline{b}^{\left(I_{l}\right)}, \bar{b}^{\left(I_{l}\right)}\right]=\left\{(U, \ell) \in \mathcal{U} \times \mathcal{L}: \mathbf{B}_{a}^{\left(I_{l}\right)}\left[U^{\prime} \ell\right]^{\prime} \leq \mathbf{B}_{b}^{\left(I_{l}\right)}\right\}$ with $\mathbf{B}_{a}^{\left(I_{l}\right)}=\left[\mathbf{I}_{m T+1}, \quad-\mathbf{I}_{m T+1}\right]^{\prime}$ and $\mathbf{B}_{b}^{\left(I_{l}\right)}=$ $\left[\bar{b}^{\left(I_{l}\right)^{\prime}}, \quad-\underline{b}^{\left(I_{l}\right)^{\prime}}\right]^{\prime}$, where $\mathbf{I}_{m T+1}$ is the identity matrix of $m T+1$ dimension.

The set $\Pi_{\tilde{z}}\left(\mathcal{B}_{l}\right)$ obtained by slicing $\mathcal{B}_{l}$ in correspondence of $\tilde{z}$ can be written as:

$\Pi_{\tilde{z}}\left(\boldsymbol{B}_{l}\right)=\left\{U_{w} \in \mathcal{W}:\left[\mathbf{B}_{a}^{\left(I_{l}\right)}\right]_{w} U_{w} \leq \mathbf{B}_{b}^{\left(I_{l}\right)}-\left[\mathbf{B}_{a}^{\left(I_{l}\right)}\right]_{v} \tilde{z}\right\}$ 


$$
= \begin{cases}{\left[\underline{b}_{w}^{\left(I_{l}\right)}, \bar{b}_{w}^{\left(I_{l}\right)}\right]} & \text { if } \tilde{v} \in\left[\underline{b}_{v}^{\left(I_{l}\right)}, \bar{b}_{v}^{\left(I_{l}\right)}\right] \\ \emptyset & \text { otherwise, }\end{cases}
$$

where we denote with $U_{w}$ the column vector obtained by extracting from $U$ the elements that correspond to the disturbance $\{w(k)\}_{k=0}^{T-1}$, and, for ease of notation, we use $\underline{b}_{w}^{\left(I_{l}\right)}$ and $\bar{b}_{w}^{\left(I_{l}\right)}$ for the vectors obtained extracting the scalar elements of $\underline{b}^{\left(I_{l}\right)}$ and $\bar{b}^{\left(I_{l}\right)}$ that correspond to $\{w(k)\}_{k=0}^{T-1}$.

If the sliced box $\Pi_{\tilde{z}}\left(\mathcal{B}_{l}\right)$ is non-empty, we next prove that a value for $\tilde{z}$ that makes the set-containment condition (21) hold can be found via a linear feasibility test.

Proposition 2: Let $\Pi_{\tilde{z}}\left(\mathcal{B}_{l}\right) \neq \emptyset$. Then, condition $\Pi_{\tilde{z}}\left(\mathcal{B}_{l}\right) \subseteq \Pi_{\tilde{z}}\left(\boldsymbol{S} \boldsymbol{p}_{l}\right)$ is satisfied if and only if:

$$
\exists \tilde{z} \in\left[\underline{b}_{z}^{\left(I_{l}\right)} \bar{b}_{z}^{\left(I_{l}\right)}\right]: \quad\left[\mathbf{S}_{a}^{\left(I_{l}\right)}\right]_{z} \tilde{z} \leq \mathbf{S}_{b}^{\left(I_{l}\right)}-\mathcal{L}^{\left(I_{l}\right)},
$$

where

$$
\mathcal{L}^{\left(I_{l}\right)}=\frac{1}{2}\left[\mathbf{S}_{a}^{\left(I_{l}\right)}\right]_{w}\left(\bar{b}_{w}^{\left(I_{l}\right)}+\underline{b}_{w}^{\left(I_{l}\right)}\right)+\frac{1}{2}\left|\left[\mathbf{S}_{a}^{\left(I_{l}\right)}\right]_{w}\right|\left(\bar{b}_{w}^{\left(I_{l}\right)}-\underline{b}_{w}^{\left(I_{l}\right)}\right) .
$$

Proof: Condition $\Pi_{\tilde{z}}\left(\boldsymbol{B}_{l}\right) \subseteq \Pi_{\tilde{z}}\left(\mathcal{S} \boldsymbol{p}_{l}\right)$ is equivalent to:

$$
\left[\mathbf{S}_{a}^{\left(I_{l}\right)}\right]_{w} U_{w}+\left[\mathbf{S}_{a}^{\left(I_{l}\right)}\right]_{z} \tilde{z} \leq \mathbf{S}_{b}^{\left(I_{l}\right)}, \quad \forall U_{w} \in\left[\underline{b}_{w}^{\left(I_{l}\right)}, \bar{b}_{w}^{\left(I_{l}\right)}\right],
$$

which holds if and only if:

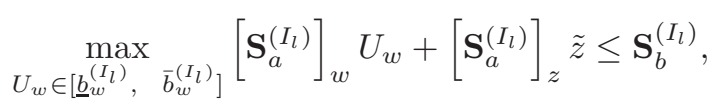

where the max operator is meant row-wise. Through the following change of variable:

$$
U_{w}=\mu_{U}+Q,
$$

where $\mu_{U}=\frac{1}{2}\left(\bar{b}_{w}^{\left(I_{l}\right)}+\underline{b}_{w}^{\left(I_{l}\right)}\right)$ and

$$
Q \in \mathcal{Q}=\left[-\frac{1}{2}\left(\bar{b}_{w}^{\left(I_{l}\right)}-\underline{b}_{w}^{\left(I_{l}\right)}\right), \quad \frac{1}{2}\left(\bar{b}_{w}^{\left(I_{l}\right)}-\underline{b}_{w}^{\left(I_{l}\right)}\right)\right],
$$

problem (23) is rewritten as:

$$
\max _{Q \in \mathcal{Q}}\left[\mathbf{S}_{a}^{\left(I_{l}\right)}\right]_{w} Q+\left[\mathbf{S}_{a}^{\left(I_{l}\right)}\right]_{w} \mu_{U}+\left[\mathbf{S}_{a}^{\left(I_{l}\right)}\right]_{z} \tilde{z} \leq \mathbf{S}_{b}^{\left(I_{l}\right)}
$$

The worst case of the term on the left hand side of (24) is found on a vertex of $\mathcal{Q}$, and its value is given by:

$$
\max _{Q \in \mathcal{Q}}\left[\mathbf{S}_{a}^{\left(I_{l}\right)}\right]_{w} Q=\left|\left[\mathbf{S}_{a}^{\left(I_{l}\right)}\right]_{w}\right| \frac{\bar{b}_{w}^{\left(I_{l}\right)}-\underline{b}_{w}^{\left(I_{l}\right)}}{2}
$$

Plugging (25) in (24) concludes the proof.

The feasibility test (22) applies to those switching sequences such that $\Pi_{\tilde{z}}\left(\boldsymbol{B}_{l}\right)$ is not empty. For those ones such that $\Pi_{\tilde{z}}\left(\boldsymbol{B}_{l}\right)$ is empty, $\Pi_{\tilde{z}}\left(\mathcal{B}_{l}\right) \subseteq \Pi_{\tilde{z}}\left(\boldsymbol{S} \boldsymbol{p}_{l}\right)$ is trivially satisfied. To account for this, we will next encode the condition

$$
\begin{aligned}
& \Pi_{\tilde{z}}\left(\boldsymbol{B}_{l}\right) \subseteq \Pi_{\tilde{z}}\left(\mathcal{S} \boldsymbol{p}_{l}\right) \Leftrightarrow \\
& \begin{cases}{\left[\mathbf{S}_{a}^{\left(I_{l}\right)}\right]_{z} \tilde{z} \leq \mathbf{S}_{b}^{\left(I_{l}\right)}-\mathcal{L}^{\left(I_{l}\right)},} & \tilde{z} \in\left[\underline{b}_{z}^{\left(I_{l}\right)}, \bar{b}_{z}^{\left(I_{l}\right)}\right] \\
\text { trivially satisfied, } & \tilde{z} \notin\left[\underline{b}_{z}^{\left(I_{l}\right)}, \bar{b}_{z}^{\left(I_{l}\right)}\right]\end{cases}
\end{aligned}
$$

within a mixed integer linear feasibility test.

Fix a switching sequence $I_{l}$ : the constraint $\tilde{z} \in$
$\left[\underline{b}_{z}^{\left(I_{l}\right)}, \bar{b}_{z}^{\left(I_{l}\right)}\right]$ that $\tilde{z}$ belongs to the box $\mathcal{B}_{l}$ can be expressed as

$$
\mathbf{T}_{a} \tilde{z} \leq \mathbf{T}_{b}^{\left(I_{l}\right)},
$$

where $\mathbf{T}_{a}=\left[\mathbf{I}_{m_{v} T+1},-\mathbf{I}_{m_{v} T+1}\right]^{\prime}$ and $\mathbf{T}_{b}^{\left(I_{l}\right)}=\left[\bar{b}_{z}^{\left(I_{l}\right)^{\prime}}-\right.$ $\left.\underline{b}_{z}^{\left(I_{l}\right)^{\prime}}\right]^{\prime}$. We now introduce $2\left(m_{v} T+1\right)$ binary variables $\sigma_{j}^{\left(I_{l}\right)}$, $j=\left\{1, \ldots, 2\left(m_{v} T+1\right)\right\}$, one per each row of (26), defined by:

$$
\sigma_{j}^{\left(I_{l}\right)}=1 \Leftrightarrow \mathbf{T}_{a, j} \tilde{z} \leq \mathbf{T}_{b, j}^{\left(I_{l}\right)} .
$$

Equation (27) can be translated via the big- $M$ technique (see [24]) into the following linear inequalities :

$$
\left\{\begin{array}{l}
\mathbf{T}_{a, j} \tilde{z}-\mathbf{T}_{b, j}^{\left(I_{l}\right)}+\bar{V}_{j}^{\left(I_{l}\right)} \sigma_{j}^{\left(I_{l}\right)} \leq \bar{V}_{j}^{\left(I_{l}\right)} \\
-\mathbf{T}_{a, j} \tilde{z}+\mathbf{T}_{b, j}^{\left(I_{l}\right)}+\underline{V}_{j}^{\left(I_{l}\right)} \sigma_{j}^{\left(I_{l}\right)} \leq 0
\end{array},\right.
$$

where $\bar{V}_{j}^{\left(I_{l}\right)}$ and $\underline{V}_{j}^{\left(I_{l}\right)}$ are given by

$$
\begin{aligned}
\bar{V}_{j}^{\left(I_{l}\right)} & =\max _{\tilde{z}} \mathbf{T}_{a, j} \tilde{z}-\mathbf{T}_{b, j}^{\left(I_{l}\right)} \\
\underline{V}_{j}^{\left(I_{l}\right)} & =\min _{\tilde{z}} \mathbf{T}_{a, j} \tilde{z}-\mathbf{T}_{b, j}^{\left(I_{l}\right)}
\end{aligned}
$$

and can be computed by using the same technique as in Proposition 2. In order to make the constraint (22) trivially satisfied when $\tilde{z} \notin\left[\underline{b}_{z}^{\left(I_{l}\right)}, \bar{b}_{z}^{\left(I_{l}\right)}\right]$, we can now exploit the binary variables defined in (27) and write the following constraint:

$$
\left[\mathbf{S}_{a}^{\left(I_{l}\right)}\right]_{z} \tilde{z}-\mathbf{S}_{b}^{\left(I_{l}\right)}+\mathcal{L}^{\left(I_{l}\right)} \leq \mathbf{Z}^{\left(I_{l}\right)} \mathbf{1}_{2\left(m_{v} T+1\right)}-\mathbf{Z}^{\left(I_{l}\right)} \sigma^{\left(I_{l}\right)},
$$

where $\sigma^{\left(I_{l}\right)}$ is a column vector obtained by stacking all $\sigma_{j}^{\left(I_{l}\right)}$ variables, $\mathbf{1}_{2\left(m_{v} T+1\right)}$ is a column vector of $2\left(m_{v} T+1\right)$ ones, and each column $[\mathbf{Z}]^{j}$ of $\mathbf{Z}$ is defined by:

$$
[\mathbf{Z}]^{j}=\max _{\tilde{z}}\left[\mathbf{S}_{a}^{\left(I_{l}\right)}\right]_{z} \tilde{z}-\mathbf{S}_{b}^{\left(I_{l}\right)}+\mathcal{L}^{\left(I_{l}\right)},
$$

where the max operator is to be interpreted row-wise. Again, (29) can be tackled by means of the same technique used in Proposition 2. Equation (28) becomes tight only if all the $\sigma_{t}^{\left(I_{l}\right)}, t=1, \ldots, 2\left(m_{v} T+1\right)$, are equal to 1 , which is equivalent to $\tilde{z} \in\left[\underline{b}_{z}^{\left(I_{l}\right)}, \bar{b}_{z}^{\left(I_{l}\right)}\right]$ (see (27)).

Finally, we can setup the following MILP problem:

$$
\min _{\tilde{z}=(\tilde{\boldsymbol{v}}, \tilde{\ell}) \in \mathcal{V} \times \mathcal{L},\left\{\sigma^{I_{l}}\right\}_{I_{l} \in \mathrm{SS}}} \tilde{\ell}
$$

subject to:

$$
\begin{aligned}
& \mathbf{T}_{a, j} \tilde{z}-\mathbf{T}_{b, j}^{\left(I_{l}\right)}+\bar{V}_{j}^{\left(I_{l}\right)} \sigma_{j}^{\left(I_{l}\right)} \leq \bar{V}_{j}^{\left(I_{l}\right)}, j=1, \ldots, 2\left(m_{v} T+1\right) \\
& -\mathbf{T}_{a, j} \tilde{z}+\mathbf{T}_{b, j}^{\left(I_{l}\right)}+\underline{V}_{j}^{\left(I_{l}\right)} \sigma_{j}^{\left(I_{l}\right)} \leq 0, j=1, \ldots, 2\left(m_{v} T+1\right) \\
& {\left[\mathbf{S}_{a}^{\left(I_{l}\right)}\right]_{z} \tilde{z}+\mathbf{Z}^{\left(I_{l}\right)} \sigma^{\left(I_{l}\right)} \leq \mathbf{S}_{b}^{\left(I_{l}\right)}-\mathcal{L}^{\left(I_{l}\right)}+\mathbf{Z}^{\left(I_{l}\right)} \mathbf{1}_{2\left(m_{v} T+1\right)}}
\end{aligned}
$$

\section{$\forall I_{l} \in \mathbf{S S}$.}

Note that the obtained solution is suboptimal since an overapproximation of the reach-sets has been adopted. However, if feasible, problem (30) returns a control input sequence $\tilde{v}^{\star}$ and an upper bound $\tilde{\ell}^{\star}$ on the cost that are feasible for the original problem (8), thus entailing that $\tilde{v}^{\star}$ enforces 
the state constraints robustly over the set $\mathcal{W}$ of disturbance realizations of predefined probability $1-\epsilon$, despite of the fact that different disturbance realizations may activate a different switching sequence.

Supposedly, problem (30) has to be solved for the admissible switching sequences only. In practice, there is no need of predetermining which sequences are admissible (which can be done via MILP as shown in [14]). Indeed, a nonadmissible switching sequence $I_{l}$ is automatically discarded when the computation of the outer box of $\mathcal{A}_{l}$ is infeasible.

The complexity of the MILP problem (30) is basically dictated by the number of involved integer variables. This number grows exponentially with the time horizon length $T$ since the number of switching sequences grows exponentially with time. If we fix $T$ and look at the dependence in the number of switching sequences, then for each switching sequence we have that, in the worst case, $2\left(m_{v} T+1\right)$ binary variables for each box are needed. In practice this number is always much smaller, since each binary variable is associated with the boundary of a box and it is often the case that a boundary is shared by multiple boxes. Note that the computational complexity does not depend on the state space dimension. This feature makes our approach particularly attractive for problems with a large state space, yet with a limited number of modes.

\section{CONCLUSIONS}

In this paper, we presented a data-driven approach to optimal control input design for a discrete time PWA system affected by a possibly unbounded stochastic disturbance subject to state and actuation constraints.

We adopted a chance-constrained approach for the optimal control problem formulation and propose a solution resting on the integration of the scenario approach to stochastic optimization and robust control for PWA systems.

Scalability issues of the resulting MILP problem could be addressed by applying model reduction techniques or the joint use of classical MILP solvers and techniques borrowed from theoretical computer science, like Boolean satisfiability problem solvers (SAT), so as to improve the performance of Branch \& Bound algorithms for MILP programs (see [25]).

Another interesting direction of research that we are currently pursuing is the extension of the approach to the class of nonlinear systems by suitably approximating their dynamics with a PWA one and accounting for the error as a bounded mode-dependent disturbance.

\section{REFERENCES}

[1] J. Lunze and F. Lamnabhi-Lagarrigue, Handbook of hybrid systems control: theory, tools, applications. Cambridge University Press, 2009.

[2] E. Asarin, T. Dang, and A. Girard, "Hybridization methods for the analysis of nonlinear systems." Acta Informatica, vol. 43, no. 7, pp. 451-476, 2007.

[3] A. Girard and S. Martin, "Synthesis for constrained nonlinear systems using hybridization and robust controllers on simplices," IEEE Transactions on Automatic Control, vol. 57, no. 4, pp. 1046-1051, 2012. [Online]. Available: http://dx.doi.org/10.1109/TAC. 2011.2168874
[4] P. Prabhakar and M. García Soto, "Hybridization for stability analysis of switched linear systems," in 19th International Conference on Hybrid Systems: Computation and Control, ser. HSCC '16. New York, NY, USA: ACM, 2016, pp. 71-80. [Online]. Available: http://doi.acm.org/10.1145/2883817.2883840

[5] S. Bak, S. Bogomolov, T. A. Henzinger, T. T. Johnson, and P. Prakash, "Scalable static hybridization methods for analysis of nonlinear systems," in 19th Intl. Conf. on Hybrid Systems: Computation and Control (HSCC 2016). ACM, Apr. 2016.

[6] J. L. Villa, M. Duque, A. Gauthier, and N. Rakoto-Ravalontsalama, "Translating pwa systems into mld systems," in 2004 IEEE International Symposium on Intelligent Control, Sept 2004, pp. 37-42.

[7] A. Bemporad, "Efficient conversion of mixed logical dynamical systems into an equivalent piecewise affine form," IEEE Transactions on Automatic Control, vol. 49, no. 5, pp. 832-838, May 2004.

[8] A. Bemporad and M. Morari, "Control of systems integrating logic, dynamics, and constraints," Automatica, vol. 35, no. 3, pp. 407-427, 1999.

[9] W. Heemels, B. D. Schutter, and A. Bemporad, "Equivalence of hybrid dynamical models," Automatica, vol. 37, no. 7, pp. 1085 - 1091, 2001. [Online]. Available: http://www.sciencedirect.com/ science/article/pii/S0005109801000590

[10] K. Margellos, P. Goulart, and J. Lygeros, "On the road between robust optimization and the scenario approach for chance constrained optimization problems," IEEE Transactions on Automatic Control, vol. 59, no. 8, pp. 2258-2263, Aug 2014.

[11] G. Calafiore and M. Campi, "Uncertain convex programs: Randomized solutions and confidence levels," Mathematical Programming, vol. 102, no. 1, pp. 25-46, 2005.

[12] M. Campi and S. Garatti, "The exact feasibility of randomized solutions of uncertain convex programs," SIAM Journal on Optimization, vol. 19 , no. 3, pp. 1211-1230, 2008 .

[13] M. Campi, S. Garatti, and M. Prandini, "The scenario approach for systems and control design," Annual Reviews in Control, vol. 33, no. 2, pp. 149-157, 2009.

[14] R. Vignali, "Automatic verification and input design for dynamical systems: an optimization-based approach to the detection of noninfluential inputs," Ph.D. dissertation, Politecnico di Milano, Milano, Italy, 2015.

[15] J. Löfberg, Minimax approaches to robust model predictive control. Linköping University Electronic Press, 2003, vol. 812.

[16] M. P. Silva, L. Pina, A. Bemporad, M. A. Botto, and J. S. da Costa, "Robust optimal control of linear hybrid systems: an MLD approach," in Proc. 6th Portuguese Conference on Automatic Control, 2004, pp. 208-213.

[17] M. P. Silva, A. Bemporad, M. A. Botto, and J. S. da Costa, "Optimal control of uncertain piecewise affine/mixed logical dynamical systems," in European Control Conference (ECC), 2003. IEEE, 2003, pp. 1573-1578.

[18] G. M. Ziegler, Lectures on polytopes. Springer Science \& Business Media, 2012, vol. 152.

[19] L. Deori, S. Garatti, and M. Prandini, "Trading performance for state constraint feasibility in stochastic constrained control: A randomized approach," Journal of the Franklin Institute, vol. 354, no. 1, pp. 501 - 529, 2017. [Online]. Available: http://www.sciencedirect.com/science/article/pii/S0016003216303799

[20] A. Prèkopa, Stochastic Programming. Boston, MA: Kluwer, 1995.

[21] E. Cinquemani, M. Agarwal, D. Chatterjee, and J. Lygeros, "Convexity and convex approximations of discrete-time stochastic control problems with constraints," Automatica, vol. 47, no. 9, pp. 2082-2087, 2011.

[22] R. Vignali and M. Prandini, "Optimal robust control of uncertain piecewise affine systems subject to reachability specifications," 2016 , submitted.

[23] F. D. Torrisi, "Modeling and reach-set computation for analysis and optimal control of discrete hybrid automata," Ph.D. dissertation, Diss., Technische Wissenschaften ETH Zürich, Nr. 15064, 2003, 2003.

[24] A. Bemporad, G. Ferrari-Trecate, and M. Morari, "Observability and Controllability of Piecewise Affine and Hybrid Systems," IEEE Transactions on Automatic Control, vol. 45, no. 10, pp. 1864-1876, 2000.

[25] A. Bemporad and N. Giorgetti, "Logic-based solution methods for optimal control of hybrid systems," IEEE Transactions on Automatic Control, vol. 51, no. 6, pp. 963-976, 2006. 